\title{
Optimization of Processing Parameters for Particle Filtration Efficiency of Polypropylene Melt-blown Fabric
}

\author{
Taijun Jiang ${ }^{1,2}$, Guangsheng Zeng ${ }^{1 *}$, Can $\mathrm{Hu}^{1}$, Cong Meng ${ }^{1}$, and Yi Chen ${ }^{1}$ \\ ${ }^{1}$ Hunan Key Laboratory of Biomass Fiber Functional Materials, Hunan International Scientific and Technological Innovation \\ Cooperation Base of Biomass Fiber Materials and Application, School of Urban and Environmental Science, \\ Hunan University of Technology, Zhuzhou 412007, China \\ ${ }^{2}$ Dongguan Mingfeng Packaging Corp. Ltd., Dongguan 523049, China
}

(Received June 8, 2020; Revised July 13, 2020; Accepted July 17, 2020)

\begin{abstract}
Polypropylene melt-blown fabric as the core filter materials plays an important role in manufacturing civil and medical epidemic prevention products. The aim of this study was to optimize and control the particle filtration efficiency and apparent surface quality of the melt-blown fabric from the perspectives of electret masterbatch composition and processing parameters. Magnesium stearate and fumed silica were used as the electret to manufacture polypropylene melt-blown fabric. The layout and charge voltage of the electret device was taken as the variables to optimize and improve the particle filtration efficiency and bulkiness coefficient of the fabric. The temperature of the barrel and die was controlled to enhance the surface quality and corresponding particle filtration efficiency. The results showed that a combination of organic magnesium stearate and inorganic fumed silica could obtain a balance between particle filtration efficiency and smooth processing without being jammed. Increasing the charge voltage and rearranging the position of the charge wires could significantly enhance the particle filtration efficiency. A better particle filtration efficiency and reasonable bulkiness coefficient could be obtained by controlling the diameter of the filaments by regulating the temperature of the barrel and die as well as the suction intensity. The results obtained from this study can offer valuable references in optimization of electret master batch formulation and processing parameters for melt-blown fabric production with different resin.
\end{abstract}

Keywords: Particle filtration efficiency, Polypropylene, Melt-blown fabric, Electret masterbatch, Bulkiness coefficient

\section{Introduction}

Melt-blown fabric made of polypropylene is a kind of wildly used filter materials in civil and medical masks, air conditioner, filter unit, and feminine hygiene products [1]. The porous structure of the melt-blown fabric and electrostatic interaction between fabric and particles around can bring an excellent filtration efficiency for particle pollutions [2]. Melt-blown fabric behaved as the core component in producing masks and medical protective clothing plays an important role during the world-wide coronavirus disease in 2019 and 2020. Besides the meltblown fabric have been obtaining their potential application in autos, sanitation products, household appliance, and water treatment device $[2,3]$.

Particle filtration efficiency of polypropylene melt-blown fabric is the most important properties when it was used to make protective mask or other filter materials. The particles could be divided into two kinds, listing as salt particle and oily particle. The salt particles were considered as the most important and popular factors when dealing with particle pollution in surrounding environment. The ability to prevent micron level particles entering our respiratory tract determines the quality of the melt-blown fabric. There were many solutions which could be used to enhance the particle filtration efficiency of melt-blown fabric [4,5]. Firstly, the

*Corresponding author: guangsheng_zeng@163.com small and porous structure in the fabric could prevent larger particles to pass through, however it was difficult to prevent smaller particles with the diameter of less than 3 micron to penetrate through for the limited size of porous channel and difficulty in controlling the dimensions of the melt-blown filaments during processing. There was another efficient way to improve the particle filtration efficiency which introduced electret into the formula of the melt-blown polypropylene. The static electricity on the surface of the porous fabric can adsorb smaller particles to the wall of the porous channel. Therefore, the electret masterbatch turned to be important for the quality control of particle filtration efficiency of the polypropylene melt-blown fabric. To improve the intensity of static electricity on the fabric we could store enough static electricity on the fabric and keep it last longer. Many researches have been done to investigate the effects of electret on the properties of electricity storage and particle filtration efficiency of the fabric [6,7]. Generally, the electret covers inorganic and organic ones. The inorganic electret has an excellent electricity storage capability and durability but it is apt to make the spinneret orifice jammed. While the organic electret is benefit to the spinning orifice but its electricity storage capability and durability are not satisfied. As a result, a combination of inorganic and organic electret was always used to obtain a balance between electricity storage performance and smoothness in processing. In addition, the processing parameters also have significant influence on the particle filtration efficiency and related 
performance of the fabric. However, fewer investigation can be found to explore the difference between organic and inorganic electret on particle filtration efficiency of the fabric and the effects of processing variables especially the layout of electret device, and temperature in barrel and die on the particle filtration efficiency, bulkiness coefficient, and surface quality of the fabric.

In this study we combined organic and inorganic electret to enhance the static electricity capacity and durability and investigated the effects of mechanical layout of the electret device as well as processing parameters on the particle filtration efficiency and surface quality of polypropylene melt-blown fabric. A self-assembled melt-blown fabric production was made, including adjustable functional units, such as web former and electret device. Techniques obtained from this study to improve the particle filtration efficiency and apparent quality from the perspectives of electret formula and processing parameters could offer valuable and operable suggestion and instruction for the fabrication of melt-blown fabric with different polymer resin. Further we could gain a deep exploration of the mechanism of particle filtration with the help of static electricity capacity and durability controlling measures.

\section{Experimental}

\section{Materials}

Polypropylene (PP, grade 230, with the melt flow index of $23 \pm 3$ g. $(10 \mathrm{~min})^{-1}$ manufactured by Sinopec in Maoming

Table 1. Formula of high flow polypropylene with pyrolysis method

\begin{tabular}{lc}
\hline \multicolumn{1}{c}{ Components } & Concentration (wt\%) \\
\hline PP & $96.1-98.1$ \\
DTBP & 0.4 \\
Erucyl amide & 0.2 \\
Antioxidant & 0.3 \\
Electret masterbatch & Varied from $1-3$ \\
\hline
\end{tabular}

was used as the raw material to produce high flow polypropylene used in melt-blown fabric with pyrolysis method with the help of di-t-butyl peroxide (DTBP). The dit-butyl peroxide with the grade of 101 was bought from Aksu. Additives erucyl amide and antioxidant (grade 215) was bought from local chemical shops. The antioxidant 215 consisted of tns-(2.4-di-tert-butyl)-phosphite and pentaerythritol tetrakys 3-(3,5-ditert-butyl-4-hydroxyphenyl) propionate with the mass ratio of $1: 1$. The electret masterbatch was selfmade by blending above prepared PP and electret. The electret covered magnesium stearate and fumed silica, both of which were bought from local chemical shop. The concentration of above-mentioned magnesium stearate and fumed silica was $20 \mathrm{wt} . \%$ and $12 \mathrm{wt} . \%$ respectively.

\section{Apparatus for Melt-blown Fabric}

The melt-blown fabric production line contains singlescrew extruder, screen exchanger, die, web former, electret device and wind-up rollers as depicted in Figure 1. An online vacuum drying hopper was equipped on the single screw extruder and the diameter of the screw was $65 \mathrm{~mm}$ with the length-diameter ratio of 30 . The three filter screens used within the screen exchanger were 100, 150 and 300 mesh in sequence in flow direction. A suction ventilator was installed behind the web and directly opposite to the spinneret plate of the die. The available voltage of the electret device ranged from 0 to $100 \mathrm{kV}$ and the rated current was $30 \mathrm{~mA}$. The diameter of the spinneret orifice was $0.25 \mathrm{~mm}$ and the interval between adjacent ones was $0.7 \mathrm{~mm}$. The width of the die was $600 \mathrm{~cm}$. The distance between fabric and charge wire used for electricity storage varied from $4.5 \mathrm{~cm}$ to $8 \mathrm{~cm}$.

\section{Experimental Procedures}

The melt-blown grade polypropylene was prepared with a twin-screw extruder with the screw diameter of $71.5 \mathrm{~mm}$ and length-diameter ratio of 52 . The temperature set for the extruder screw were $175^{\circ} \mathrm{C}, 185^{\circ} \mathrm{C}, 195^{\circ} \mathrm{C}, 205^{\circ} \mathrm{C}, 205^{\circ} \mathrm{C}$, $205^{\circ} \mathrm{C}, 200{ }^{\circ} \mathrm{C}, 200^{\circ} \mathrm{C}, 195^{\circ} \mathrm{C}, 190^{\circ} \mathrm{C}, 190{ }^{\circ} \mathrm{C}, 185^{\circ} \mathrm{C}$ and $185^{\circ} \mathrm{C}$ from the feeding zone to pelletizing die. Before the

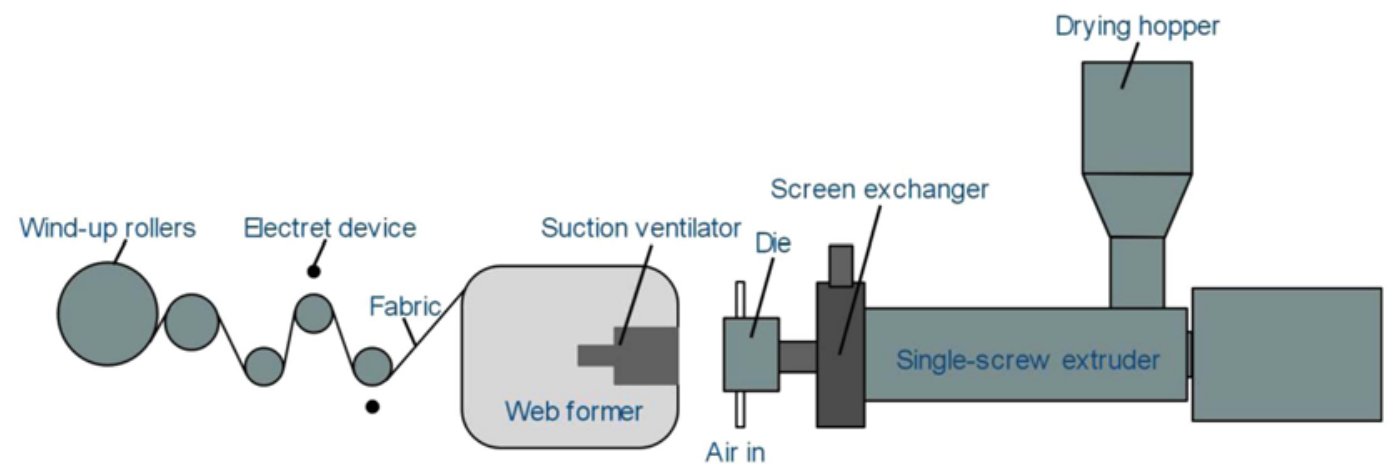

Figure 1. Structure of polypropylene melt-blown fabric production line. 
ingredients of the raw materials in Table 1 were feed into the hopper of the twin-screw extruder they must be mixed in a low-speed mixer for 0.5 hour. The melt flow index of the modified melt-blown grade polypropylene remained at $1500 \mathrm{~g} .(10 \mathrm{~min})^{-1}$ with the tolerance of $100 \mathrm{~g} .(10 \mathrm{~min})^{-1}$.

The modified polypropylene was first dried in a vacuum drying hopper for at least one hour before it entered the screws of single screw extruder. The temperature set for the single-screw extruder were $180{ }^{\circ} \mathrm{C}, 215^{\circ} \mathrm{C}, 225^{\circ} \mathrm{C}, 225^{\circ} \mathrm{C}$ respectively for each zone. And the temperature for the die was $235^{\circ} \mathrm{C}$. The temperature for the blown air was set to $276{ }^{\circ} \mathrm{C}$. The speed for the suction ventilator was set to $30 \mathrm{~Hz}$. The switch frequency for the screen exchanger was set as 6 times per hour to ensure the spinning process smooth.

\section{Particle Filtration Efficiency and Resistance Test}

The particle filtration efficiency was tested according to GB/T 32610-2016. The test was not conducted in 2 hours after the fabric was fully prepared. The temperature of the fabric must remain at room temperature during the test. The air flow rate for the test was set to $32 \pm 2 \mathrm{Liter} \cdot \mathrm{min}^{-1}$. Resistance test of the fabric was also conducted as per GB/T 32610-2016. Only sodium chloride $(\mathrm{NaCl})$ solution with the concentration of $0.9 \%$ by weight was used to produce the designed particles with various diameters during the particle filtration efficiency and resistance test in this paper. According to the standard the particle filtration efficiency of melt-blown fabric for particles with the diameter of 0.3 micro meter was taken as the valid data. The unit for resistance was pascal. The test was conducted at the relative humility of $30 \pm 10 \%$ and the temperature of $25 \pm 5^{\circ} \mathrm{C}$. The samples were placed in the above-mentioned conditions for 24 hours before the test.

Unit Fabric Weight and Average thickness Test

The unit weight of fabric was measured by weighting the

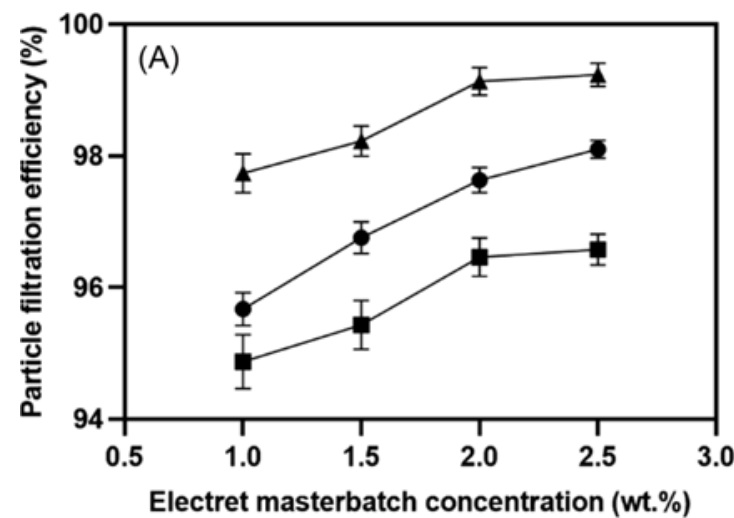

circular fabric samples cut from the online web with a sampling device. The area of the circular fabric samples was $100 \mathrm{~cm}^{2}$. The unit weight of the fabric was measured in the unit of gram $\cdot \mathrm{m}^{-2}$, which can be calculated with sample weight. Six repeated samples were collected for the test to obtain an average value. The average thickness of the fabric was obtained by averaging six thickness value at different point within one circular fabric. The ratio of unit fabric weight and average thickness could be used to characterize the bulkiness of the fabric, which was defined as bulkiness coefficient with the unit of $1000 \mathrm{~g} \cdot \mathrm{m}^{-3}$.

\section{Number of Crystal Point Calculation}

Number of crystal point on the fabric was counted within above mentioned circular fabric samples. The unit could be defined as $(100 \mathrm{~cm})^{-2}$. Three repeated samples were collected for each test. Those samples for crystal point calculation were collected along the width of the web.

\section{Results and Discussion}

\section{Electret Masterbatch}

Static electricity on polypropylene melt-blown fabric can significantly enhance the particle filtration efficiency. However, it will gradually attenuate with time especially at higher temperature and moisture content. As a result, the durability of presence of static electricity becomes important during manufacturing of such melt-blown fabric and further subsequent products. Two kind of electrets including inorganic and organic were collected to investigate the effects of electret types and their concentration on particle filtration efficiency. In order to find out the best ratio of the combined electrets magnesium stearate and fumed silica the particle filtration efficiency and its attenuation rate were considered in the experiments.

Figure 2 compared the particle filtration efficiency of

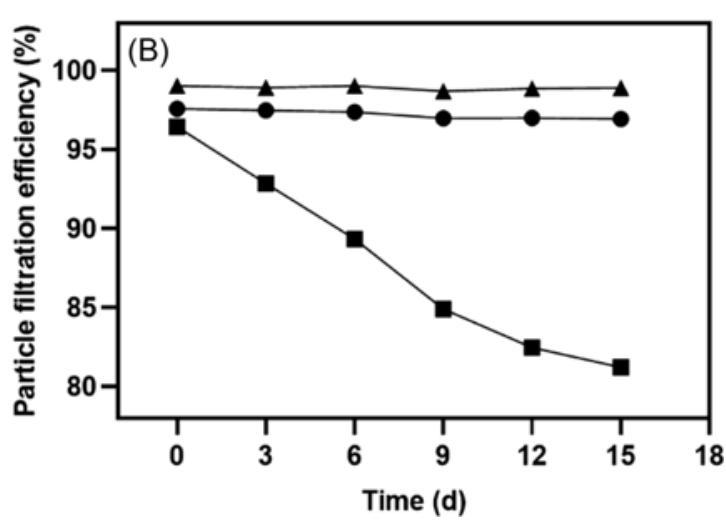

Figure 2. Particle filtration efficiency of polypropylene melt-blown fabric with different electret (magnesium stearate and fumed silica) at different concentration. The symbols $\mathbf{\Delta} \mathbf{\bullet} \bullet$ present fumed silica, magnesium stearate, and combined fumed silica/magnesium stearate with the mass ratio of 3/5; (A) effects of types of electret and their concentration on PFE and (B) attenuation of PFE. The electret masterbatch concentration used in (B) was 2 wt.\%. 
polypropylene melt-blown fabric with different types and concentration of electret and illustrated the attenuation of PFE for different electret. As is depicted, the particle filtration efficiency of the fabric increased with the concentration of any kind of electret masterbatch, but the inorganic electret fumed silica exhibited better effect than the organic electret magnesium stearate. However, the agglomeration of fumed silica filler would occur and further result in frequent jam in the spinneret orifice. And the organic magnesium stearate would be melted as fluid during processing and never cause a jam in the spinneret orifice. It could be concluded from Figure 2(B) that the organic electret could not hold the static electricity for a long time while the durability of the static electricity would improve significantly with the addition of inorganic fumed silica. The
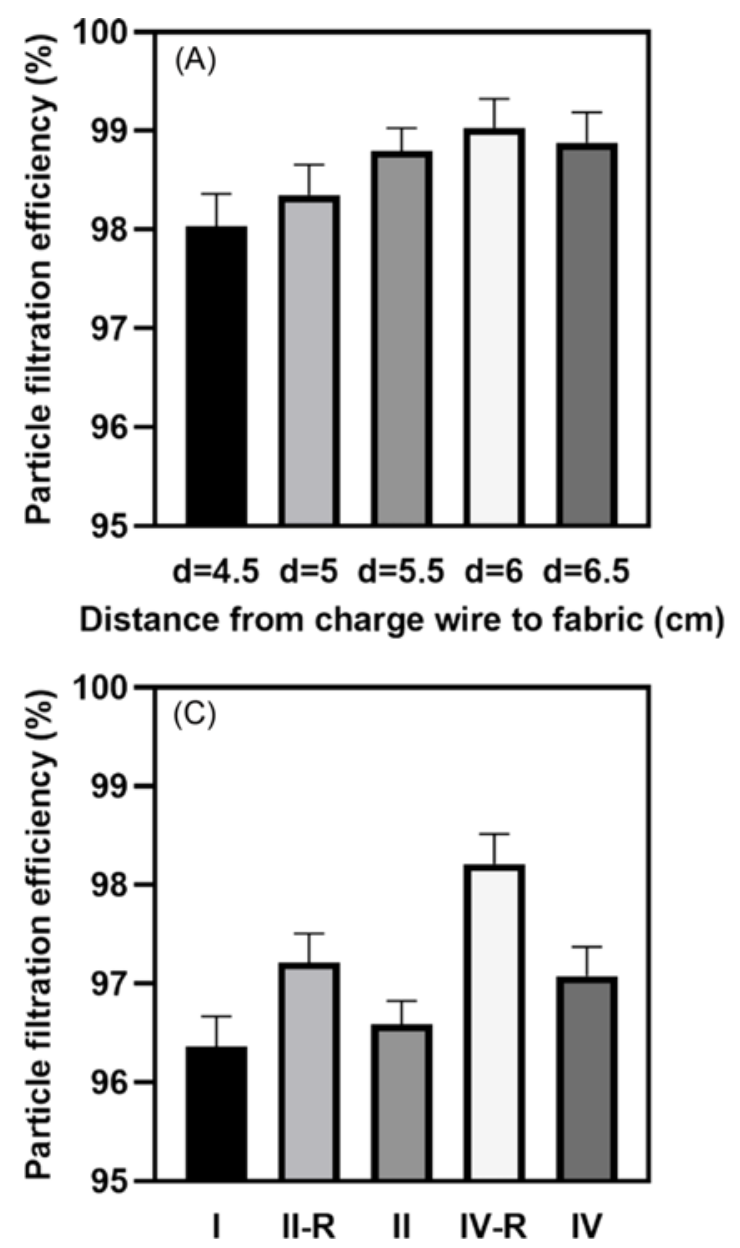

organic electret can only capture the electron on the surface and in certain depth below the surface and would easily get neutralized by external negative electron especially at higher temperature and moisture content. While the inorganic electret has an excellent storage capacity for electron and the electron would seldom vanish even at high temperature and moisture content. A combination of inorganic fumed silica and organic magnesium stearate can obtain a balance between electricity storage capacity and durability for the fabric.

\section{Electret Charge Parameters}

Static electricity on the melt-blown fabric plays an important part in improving the particle filtration efficiency of the fabric. The static electricity can be obtained by
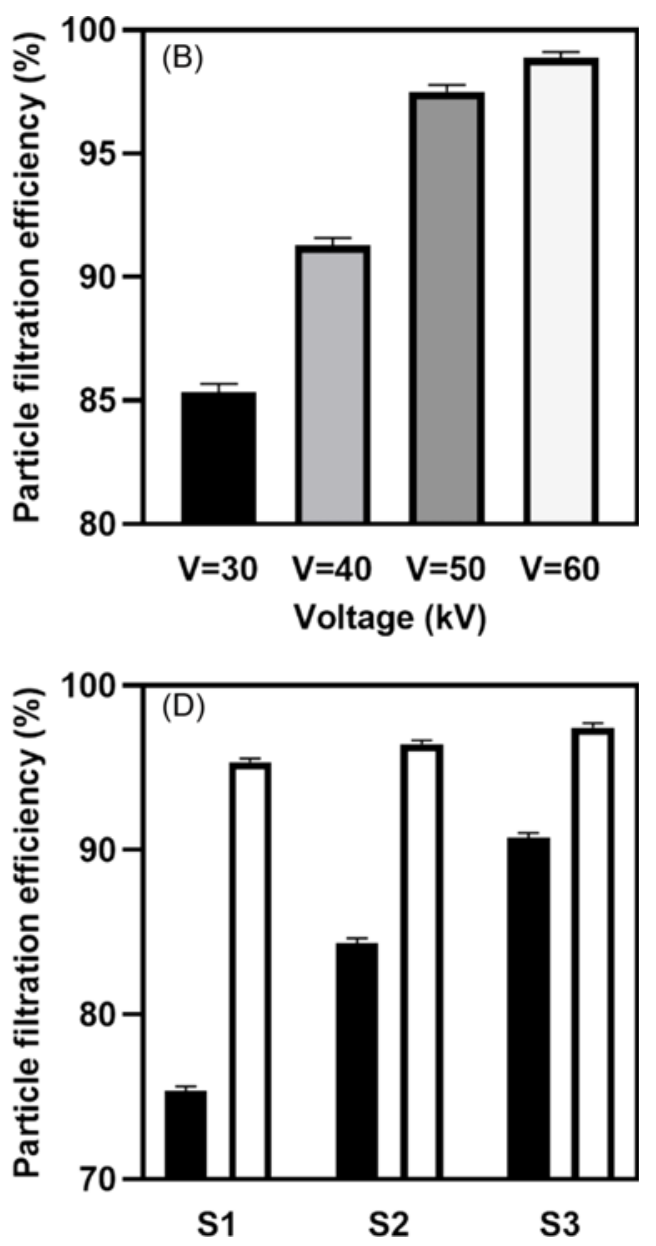

Figure 3. Particle filtration efficiency of polypropylene melt-blown fabric at different processing conditions; (A) PFE of melt-blown fabric at different charge distance from the charge wire to the fabric. The charge voltage remained at $50 \mathrm{kV}$ and two charge wires were placed at both sides of the fabric, (B) PFE of melt-blown fabric at different charge voltage. The distance from charge wire to the fabric remained at 6 $\mathrm{cm}$ and two charge wires were placed at both sides of the fabric, (C) PFE of melt-blown fabric treated by various charge wires at different position. The number I-IV represents the charge wire used and the symbol -R indicates the charge wires were placed at both sides of the fabric, and (D) PFE of melt-blown fabric after secondary electret charging treatment. The secondary treatments were conducted under the charge voltage of $50 \mathrm{kV}$ with charge wire distance to the fabric of $6 \mathrm{~cm}$. The samples used for the secondary electret charging treatments contained electret masterbatch with organic magnesium stearate only. 
charging the fabric containing electret masterbatch with electret device which can produce high voltage electric field. There are many variables which can influence the electricity storage capacity and durability, including charge voltage, current, arrangement of the charge wire [5]. Above mentioned influencing factors would affect the intensity of electron delivery from the wire to the electret on or in the fabric and the electromagnetic field which would polarize the electret to be polar at microstructural level.

The effects of related variables on the particle filtration efficiency of the fabric are illustrated in Figure 3. From Figure 3(A) we could find that the particle filtration efficiency of the fabric increased with the distance from charge wire to fabric and began to decrease at the turning point of $6 \mathrm{~cm}$. Figure 3(B) showed that the particle filtration efficiency of fabric increased with the charging voltage and it exhibited a higher rising speed at initial increasing stage of charging voltage. Figure $3(\mathrm{C})$ revealed that the particle filtration efficiency of fabric could be related to the number of charge wires and their position. More charge wires above the fabric could produce a better particle filtration efficiency. Particle filtration efficiency of fabric treated by charging at both sides behaved a better performance than that at the same side. As is known the static electricity on the fabric would attenuate with time especially for organic electret solely filled polypropylene compound. The effect of secondary electret charging treatments on the particle filtration efficiency of the fabric could be found in Figure 3(C). It could be found that the secondary electret charging for the organic electret masterbatch filled polypropylene melt-blown fabric could greatly improve the particle filtration efficiency by $26.45 \%$. And the increment in particle filtration efficiency of fabric was to a great extend determined by the initial particle filtration efficiency value before the secondary electret charging treatment. The lower initial value in particle filtration efficiency of the fabric could gain a better and obvious improvement.

Electret charge was a popular and effective way to
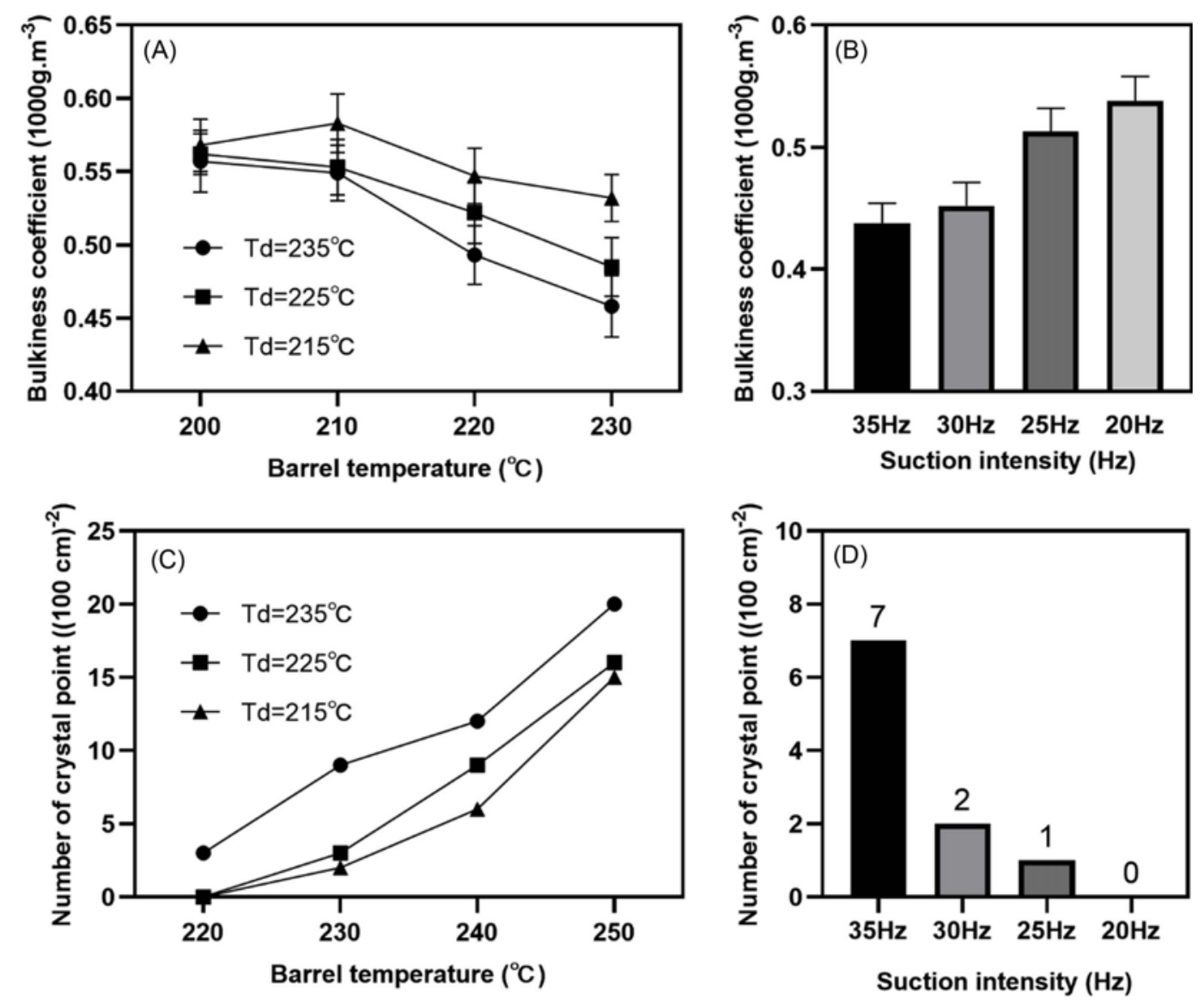

Figure 4. Bulkiness coefficient and number of crystal point of polypropylene melt-blown fabric at different processing temperature and suction intensity; (A) variation of bulkiness coefficient with barrel temperature. Td refers to the temperature of the die, (B) effect of suction intensity on number of crystal point, (C) variation of number of crystal point with barrel temperature, (D) effect of suction intensity on number of crystal point. The barrel temperature herein referred to that at zone 3 and 4 (end part of the screw where melting process occurs) and the temperature at the other two zones remained the same for convenience. 
enhance the particle filtration efficiency of the melt-blown fabric and many variables for the electret device and processing parameters would affect the effectiveness and efficiency, including charging voltage, distance to the fabric, and electric polarity [8]. As the polluted particles in the air contains negative electricity, positive electricity charging method was used to treat the polypropylene melt-blown fabric in this paper. The higher charging voltage and smaller charging distance could induce a larger charging current and shorter charging time for the fabric at fixed static electricity capacity [9]. The results suggested that the charging voltage would vary for different equipment where the distance from the charge wire to the fabric might be different. To make best of electret device the optimized distance from the charge wire to the fabric should be determined first and search for the best charge voltage later. The particle filtration efficiency of the fabric would be poorer if the fabric was only electret charging treated on one side. By increasing the number of charge wires and rearranging them symmetrically on both side the particle filtration efficiency of the fabric would be greatly improved. The particle filtration efficiency of fabric must be higher than $95 \%$ for most applications and it would attenuate after a few days. The experiments proved that a secondary electret charge turned out to be a significant and feasible measures to enhance the particle filtration efficiency of the fabric.

\section{Temperature and Suction Intensity}

Polypropylene must get melted thoroughly before it enters the spinneret orifice and the melt from the spinneret orifice will later be stretched by the high-speed heated wind to form fine and curved filaments at higher melt temperature.
Filaments with a lower diameter could produce tight but porous structure which was beneficial to particle filtration efficiency of the fabric. But the variation of temperature and suction intensity behind the web would cause quality problems including bulkiness coefficient and crystal point on the fabric. In order to investigate the effects of temperature and suction intensity on the bulkiness coefficient and number of crystal point per unit area we varied the temperature in barrel and die as well as suction intensity to check the quality defects. Figure 4 shows the variation of bulkiness coefficient and number of crystal point of polypropylene melt-blown fabric as a function of barrel temperature, die temperature, and suction intensity. It can be drawn from Figure 4(A) and (C) that the bulkiness coefficient of fabric decreased but the number of crystal point increased with barrel and die temperature. Figure 4(B) shows that the decrease of suction intensity could result in a rise in bulkiness coefficient, indicating the suction of the wind behind the web could promote the fabric to form compact structure. The number of crystal point on the fabric went up dramatically with the increase of suction intensity as depicted in Figure 4(D).

Barrel temperature is crucial for the polypropylene to get melted and obtain a good flowability. A slight increase in barrel and die temperature would result in significant variation of melt flow index for high flow polypropylene. And the polypropylene melt with higher melt flow index could produce extra fine filaments, where flurry fabric with a lower density could form. From the experimental manufacturing process we found that the sprayed filaments would crack when the melt temperature went too high (above $240{ }^{\circ} \mathrm{C}$ ) and the cracked filaments further aggregated
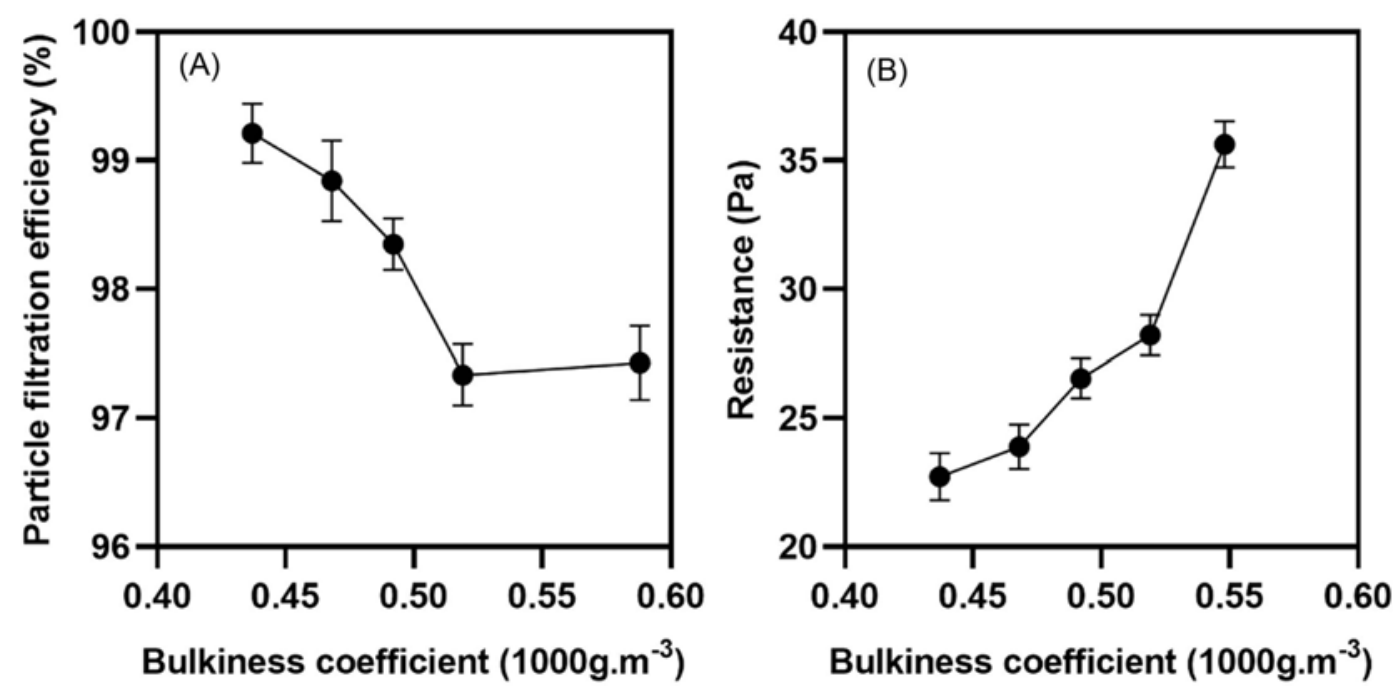

Figure 5. Relationship between particle filtration efficiency, resistance and bulkiness coefficient of polypropylene melt-blown fabric with combined electret masterbatch concentration of $2.5 \mathrm{wt} . \%$; (A) variation of PFE of polypropylene melt-blown fabric with bulkiness coefficient. Two charge wires at both side of the fabric was used. The electret charge voltage was set to $50 \mathrm{kV}$ and (B) effect of bulkiness coefficient on the resistance of the fabric. 
in the form of crystal point on the surface of the fabric. The suction ventilator behind the web could force air out across the web and helped to stretch and cool the spayed filaments. The tensile force additionally applied on the filaments would further promote the filaments to become finer and finally break down $[8,10]$. This is why the bulkiness coefficient of the fabric could decrease and the number of crystal point could go up with the suction intensity. Our findings suggest that the barrel and die temperature as well as the suction intensity should be considered simultaneously when debugging and optimizing the processing parameters for manufacturing polypropylene melt-blown fabric.

\section{Particle Filtration Efficiency and Bulkiness Coefficient}

The unit fabric weight and thickness are two important properties to evaluate the quality of polypropylene meltblown fabric and are associated with an important performance particle filtration efficiency. As mentioned above the thickness of the fabric can transferred to another parameter bulkiness coefficient. Therefore, it is necessary to figure out the relationship between particle filtration efficiency, resistance and bulkiness coefficient. Figure 5 shows the effect of bulkiness coefficient on the particle filtration efficiency and resistance respectively. As is shown in Figure 5(A), the particle filtration efficiency of the fabric decreased with bulkiness coefficient linearly from 0.42 to $0.52 \times 1000 \mathrm{~g} \cdot \mathrm{m}^{-3}$ and reached a plateau afterward. On the contrary, the resistance of the fabric increased with the bulkiness coefficient within the experimental test range. The results indicated that both particle filtration efficiency and resistance of polypropylene melt-blown fabric were related to the unit fabric weight and average thickness which were tightly monitored and controlled during the manufacturing process.

Bulkiness coefficient is a function of unit fabric weight and average thickness. The unit fabric weight is controlled by the speed of screw and web former. A higher speed of screw and lower speed of the web can result in an increase in the unit fabric weight. The polypropylene filaments with smaller diameter could form porous fabric with a higher thickness of identical structure. The polluted particles must pass through longer and porous channel to penetrate through the fabric, directly increasing particle filtration efficiency. While the longer channel would induce an increase in resistance for the air to pass through. If the speed of screw and web remained constant, the bulkiness coefficient would be affected by the temperature in barrel and die as discussed above. In this situation, the sprayed polypropylene filaments turned smaller in diameter and further caused a decrease in the size and an increase in number of porous channels with the increase in temperature in barrel and die [1]. Both of the two variations brought about an increase in particle filtration efficiency of the fabric. From the experimental results in
Figure 5(B) we can conclude that the variation of resistance are mainly caused by the increase in the number of porous channels.

\section{Conclusion}

This paper investigated the effects of electret masterbatch and processing parameters on the particle filtration efficiency, bulkiness coefficient, and surface quality of polypropylene melt-blown fabric. The results showed that the electret combined with magnesium stearate and fumed silica could brought about excellent particle filtration efficiency and smooth processing. The particle filtration efficiency of the fabric could be further improved by increasing the charge voltage and rearranging the position of the charge wires. In addition, the fabric could obtain a better particle filtration efficiency and reasonable bulkiness coefficient by controlling the diameter of the filaments through regulation of the temperature in barrel and die as well as the suction intensity. The insights obtained from the experiments in this study can offer valuable references in optimizing and controlling the performance and quality of polypropylene melt-blown fabric.

\section{Acknowledgements}

This work was sponsored by national natural science foundation of China under grant 51973056, scientific research projects of Hunan provincial department of education under grant 18A258 and 18C0524, and Hunan provincial scientific research and innovation project under grant CX20190843 and CX20190844.

\section{References}

1. Y. Pu, J. Zheng, F. Chen, Y. Long, H. Wu, Q. Li, S. Yu, X. Wang, and X. Ning, Polymers, 10, 959 (2018).

2. H. Wang, Y. Zhang, H. Gao, X. Jin, and X. Xie, Int. J. Hydrogen Energy, 41, 324 (2016).

3. J. Aszyk, P. Kubica, J. Namieśnik, A. Kot-Wasik, and A. Wasik, Anal. Chim. Acta, 1038, 67 (2018).

4. A. Brochocka, A. Zagawa, R. Panek, J. Madej, and W. Franus, Autex Res. J., 19, 312 (2019).

5. Y. Shen, S. Xia, P. Yao, R. Hugh Gong, Q. Liu, and B. Deng, Fiber. Polym., 18, 1568 (2017).

6. K. B. Im and Y. K. Hong, Text. Sci. Eng., 51, 186 (2014).

7. A. Kilic, E. Shim, and B. Pourdeyhimi, J. Electrostat., 74, 21 (2015).

8. H. Chen, H. Li, X. Ma, W. He, J. Tan, and W. Yang, Fiber. Polym., 17, 576 (2016).

9. P. P.-y. Tsai, G.-W. Qin, and C. Hassenboehler, Int. Nonwovens $J$., os-9, 8 (2000).

10. M. Guo, H. Liang, Z. Luo, Q. Chen, and W. Wei, Fiber. Polym., 17, 257 (2016). 